\section{Imaging pathophysiological changes in the lungs in IPF with xenon magnetic resonance imaging}

\author{
Jim M Wild
}

Although forced vital capacity is validated for the assessment of idiopathic pulmonary fibrosis (IPF) progression and prediction of mortality, the need for tests that are more sensitive to pathophysiological change in the lungs in IPF is well recognised for earlier diagnosis, longitudinal assessment and for better markers of therapy and prognosis. ${ }^{1}$

Imaging in IPF in clinical radiological practice currently resides with structural computed tomography. The structural changes seen in IPF on computed tomography are regionally heterogeneous and represent different aspects of the pathology and lung disease evolution. ${ }^{2}$ Computed tomography is now being used more quantitatively with image segmentation and texture analysis to classify the different structural changes. These algorithms show prognostic promise, ${ }^{3}$ but it remains to be seen how sensitive they are to disease progression. There is a possibility that manifestation of structural changes on computed tomography is too late in the disease process for effective treatment in IPF.

Magnetic resonance imaging is a modality best associated with soft tissue imaging and it does not feature in the regular diagnostic toolbox for clinical assessment of lung disease. Magnetic resonance imaging with inhaled hyperpolarised inert gases (such as ${ }^{3} \mathrm{He}$ and ${ }^{129} \mathrm{Xe}$ which are magnetically sensitive) can provide novel and diverse functional and structural information from the lungs. These methods have been brewing in magnetic resonance imaging labs for some time and a solid body of clinical research evidence now exists in a variety of different lung diseases. In a recent editorial in Thorax, the sensitivity of hyperpolarised gas ventilation magnetic resonance imaging to early obstructive airways disease in paediatric cystic fibrosis was highlighted. ${ }^{4}$

In this paper from the Duke group, ${ }^{5}$ we now see the potential for hyperpolarised ${ }^{129} \mathrm{Xe}$ magnetic resonance imaging,

Correspondence to Professor Jim M Wild, Academic Radiology, University of Sheffield, Royal Hallamshire Hospital, Sheffield S10 2JF, UK; j.m.wild@sheffield.ac.uk for assessing gas exchange and interstitial disease in the lungs in IPF. Xenon (Greek xenos-strange) is a noble gas atom, whose magnetic resonance signal can be boosted with hyperpolarisation to levels where it can be imaged in the lungs. Unlike ${ }^{3} \mathrm{He}$, ${ }^{129} \mathrm{Xe}$ is cheaper and readily available so has wider clinical potential. Xenon's solubility and unique spectral signature from its environment, either as gas in the alveoli, when dissolved in the interstitial tissue, or taken up by the red blood cells in the capillaries, make it particularly interesting for measuring diffusion limitation. Previous studies with ${ }^{129} \mathrm{Xe}$ magnetic resonance spectroscopy have focused on whole lung measurements of the dynamics of the xenon signals from the tissue, blood and airspaces, ${ }^{6}$ in order to estimate the interstitial barrier thickness and have shown differences between healthy and IPF lungs.

These approaches are extended here with a regional spectroscopic imaging technique ${ }^{5}$ giving us simultaneous structural and functional information on fibrotic change and gas exchange. Of particular interest is the increased ratio of the signal from the interstitial tissue to that from the red blood cells in the capillaries, which indicates diffusion limitation. In IPF lungs, we see a basal and peripheral distribution of the tissue-to-blood signal ratio, which is consistent with the known anatomical heterogeneity of the disease. What is also really interesting is the fact that the tissue and red blood cell signals when averaged over the whole lungs showed weak correlation with the qualitative computed tomography scores used. Furthermore, there was no clear matching of the magnetic resonance maps with structural changes on computed tomography in the patients with early fibrotic change. The method is clearly measuring different, but related aspects of the pathophysiology to structural computed tomography, in that the size of the xenon signal in a given voxel is weighted by a combination of specific ventilation, interstitial thickening, delayed gas diffusion and perfusion deficit. The correlation of the xenon indices with the carbon monoxide diffusion factor $\left(\mathrm{D}_{\mathrm{LCO}}\right)$ measured from the whole lungs corroborates this added physiological sensitivity.

The next step is to see how the regional pathophysiological sensitivity of xenon magnetic resonance imaging compares with $\mathrm{D}_{\mathrm{LCO}}$, carbon monoxide transfer coefficient, forced vital capacity and quantitative computed tomography analysis for monitoring disease progression. We could envisage an ionising radiation-free imaging examination with xenon magnetic resonance imaging used alongside promising proton magnetic resonance imaging methods ${ }^{7}$ for the longitudinal assessment of structural and functional changes in interstitial lung diseases.

\section{Competing interests None declared.}

Provenance and peer review Commissioned; internally peer reviewed.

(C) Article author(s) (or their employer(s) unless otherwise stated in the text of the article) 2018. All rights reserved. No commercial use is permitted unless otherwise expressly granted.

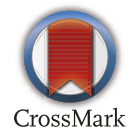

To cite Wild JM. Thorax 2018;73:1.

Published Online First 2 November 2017

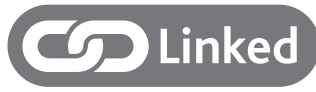

- http://dx.doi.org/10.1136/thoraxjnl-2017-210070

Thorax 2018;73:1.

doi:10.1136/thoraxjnl-2017-210861

\section{REFERENCES}

1 Wells AU. Forced vital capacity as a primary end point in idiopathic pulmonary fibrosis treatment trials: making a silk purse from a sow's ear. Thorax 2013;68:309-10.

2 Hansell DM, Goldin JG, King TE, et al. CT staging and monitoring of fibrotic interstitial lung diseases in clinical practice and treatment trials: a position paper from the Fleischner Society. Lancet Respir Med 2015;3:483-96.

3 Jacob J, Bartholmai BJ, Rajagopalan S, et al. Mortality prediction in idiopathic pulmonary fibrosis: evaluation of computer-based CT analysis with conventional severity measures. Eur Respir J 2017;49.

4 Davies JC. Visualising early lung disease in CF: the emergence of MRI. Thorax 2017;72:682.

5 Wang JM, Robertson SH, Wang Z, et al. Using hyperpolarized ${ }^{129} \mathrm{Xe}$ MRI to quantify regional gas transfer in idiopathic pulmonary fibrosis. Thorax 2018;73:21-8

6 Stewart NJ, Leung G, Norquay G, et al. Experimental validation of the hyperpolarized 129 Xe chemical shift saturation recovery technique in healthy volunteers and subjects with interstitial lung disease. Magn Reson Med. Published Online First: 8 Aug 2014. doi:10.1002/ mrm. 25400 .

7 Johnson KM, Fain SB, Schiebler ML, et al. Optimized 3D ultrashort echo time pulmonary MRI. Magn Reson Med 2013;70:1241-50. 